\title{
Prognostic Factors of Surgical Outcome after Spinous Process-Splitting Laminectomy for Lumbar Spinal Stenosis
}

\author{
Keishi Maruo, Toshiya Tachibana, Shinichi Inoue, Fumihiro Arizumi, Shinichi Yoshiya \\ Department of Orthopaedic Surgery, Hyogo College of Medicine, Nishinomiya, Japan
}

\section{Study Design: A retrospective case review.}

Purpose: To assess the clinical and radiographic outcomes and identify the predictive factors associated with poor clinical outcomes after lumbar spinous process-splitting laminectomy (LSPSL) for lumbar spinal stenosis (LSS).

Overview of Literature: LSPSL is an effective surgical treatment for LSS. Special care should be taken in patients with degenerative lumbar scoliosis (DLS).

Methods: A consecutive retrospective case review of patients undergoing LSPSL for LSS with a minimum 2-year follow-up was performed. Mild DLS and mild degenerative spondylolisthesis (DS) were included in the study. The Japanese Orthopedic Association (JOA) score and recovery rate were reviewed. Poor clinical outcome was defined as a recovery rate $<50 \%$ using Hirabayashi's method.

Results: A total of 52 patients (mean age, 72 years) met the inclusion criteria and had a mean follow-up of 2.6 years (range, 2-4.5 years). The preoperative diagnosis was LSS in 19 , DS in 19 , and DLS in 14 cases. The mean JOA score significantly increased from 14.6 to 23.2 at the final follow-up. The overall mean recovery rate was $60.1 \%$. Thirteen patients $(25 \%)$ were assigned to the poor outcome group. A higher rate of pre-existing DLS was observed in the poor outcome (poor) group (good, 15\%; poor, 62\%; $p=0.003$ ) than in the good outcome (good) group. None of the patient factors examined were associated with a poor outcome. A progression of slippage $\geq 5 \mathrm{~mm}$ was found in 8 of 24 patients (33\%) in the DS group. A progression of curvature $\geq 5^{\circ}$ was found in 5 of 14 patients (36\%) in the DLS group. The progression of scoliosis and slippage did not influence the clinical outcome.

Conclusions: The clinical and radiographic outcomes of LSPSL for LSS were favorable. Pre-existing DLS was significantly associated with poor clinical outcome.

Keywords: Lumbar spinal stenosis; Lumbar spinous process-splitting laminectomy; Degenerative lumbar scoliosis; Decompression surgery

\section{Introduction}

Surgical treatment for lumbar spinal stenosis (LSS) is widely performed, and its effectiveness has been demonstrated in prospective randomized studies [1,2]. Expan- sive laminectomy is the most commonly used surgical procedure for LSS. The potential risks of conventional laminectomy include persistent back pain due to the extensive detachment of the paraspinal muscle, trunk extensor weakness, and iatrogenic spondylolisthesis [3-7]. Less

Received Feb 1, 2015; Revised Feb 16, 2015; Accepted Feb 20, 2015

Corresponding author: Keishi Maruo

Department of Orthopaedic Surgery, Hyogo College of Medicine,

1-1 Mukogawa-cho, Nishinomiya, Hyogo 663-8501, Japan

Tel: +81-798-45-6452, Fax: +81-798-45-6453, E-mail: kmaruo@hyo-med.ac.jp 
invasive approaches and methods have been developed to preserve the posterior elements, including the lamina, spinous processes, interspinous ligaments, facet joint, and to decrease paraspinal muscle damage. Lumbar spinous process-splitting laminectomy (LSPSL) was introduced in 2005 as a less invasive technique that preserves the paravertebral muscles and posterior elements and reduces muscle atrophy and postoperative wound pain for treatment of LSS [8]. This method can easily allow a wide field of view of the central canal and lateral recess while preserving the facet joint. The LSPSL technique has been performed since 2008 at our institution for the treatment of LSS, mild degenerative spondylolisthesis (DS), and mild degenerative lumbar scoliosis (DLS). This procedure may avoid iatrogenic lumbar instability more than conventional laminectomy. However, there have been few reports regarding the clinical outcomes and radiographic evaluations after LSPSL. The objective of this study was to assess the clinical and radiographic outcomes and identify the predictive factors associated with poor clinical outcomes after LSPSL.

\section{Materials and Methods}

\section{Patient population}

In this study, a retrospective design consisting of a review of a consecutive series of patients treated with LSPSL for LSS was used. After Institutional Review Board approval, patients with LSS who had undergone LSPSL with a minimum 2-year follow-up between 2008 and 2011 were identified from a single institutional database. The diagnosis of lumbar canal stenosis was confirmed by magnetic resonance imaging findings and physical examinations, such as neurogenic claudication. Surgical treatments were considered for the patients who had persistent leg symptoms caused by cauda equine syndrome or radiculopathy. Decompression surgery was indicated for the patients without lumbar spinal instability. In cases of DLS, a Cobb angle $<30^{\circ}$, a vertebral lateral slip $<6 \mathrm{~mm}$, and the absence of foraminal stenosis were indications for decompression surgery. In cases of DS, a vertebral translation $<25 \%$ (Meyerding grade I), a change in translation $<10 \%$ on flexion and extension lateral radiographs, and a posterior disc wedge $<5^{\circ}$ on lateral radiographs were indications for decompression surgery. Microscopic bilateral decompression via a unilateral approach was generally indicated for single level lumbar stenosis and was performed at the surgeon's preference. LSPSL was mainly indicated for multilevel lumbar stenosis. Patient demographic and surgical data were obtained from medical records and operative reports.

\section{Surgical technique of LSPSL}

A 4-cm posterior midline skin incision was made between the cranial edge of the proximal spinous processes and distal spinous process (Fig. 1A, E). The posterior surface of the spinous process was identified and cut longitudinally by using a bone saw. The bottoms of the spinous processes were horizontally divided with an osteotome. The paravertebral muscles from the lamina to the medial aspect of the facet joints were exposed (Fig. 1B, F). A partial laminectomy and removal of the ligamentum flavum were performed to decompress the spinal canal using the standard technique. Subsequently, a partial facetectomy was performed to decompress the bilateral nerve roots (Fig. 1C, G). The split spinous process was reconstructed by suturing with a 1-0 nylon (Fig. 1D, H). Preoperative and postoperative computed tomography showed satisfactory decompression with preservation of the facet joint (Fig. 2).

\section{Clinical assessment}

The Japanese Orthopedic Association (JOA) score, recovery rate, and complications were reviewed to evaluate clinical outcomes. Hirabayashi's method was used to calculate the recovery rate of the JOA score [9]. The poor clinical outcome group consisted of patients with a recovery rate $<50 \%$. The patient factors of age, gender, preoperative JOA score, preoperative distance of intermittent claudication (IC), preoperative motor weakness, urinary dysfunction, duration of symptoms, and number of decompression levels were assessed to determine any associations with poor clinical outcomes. Radiographic measures included preoperative and postoperative lumbar lordosis (LL), Cobb angle, and percentage of slip. The influence of radiographic changes, such as progression of slippage ( $\geq 5 \%)$ and progression of scoliosis $\left(\geq 5^{\circ}\right)$, were evaluated by assessing the recovery rates. The clinical outcomes of the LSS group, DLS group, and DS group were compared. 


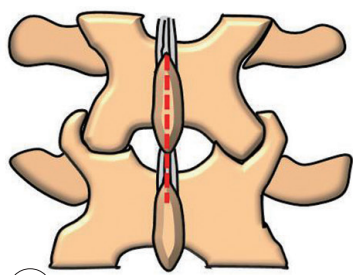

(A)

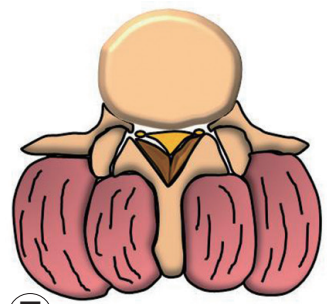

(E)

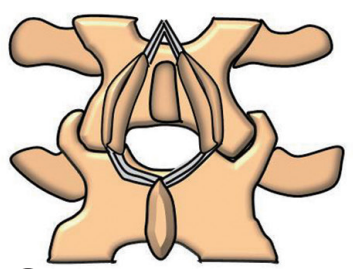

(B)

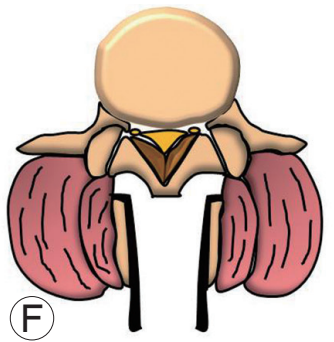

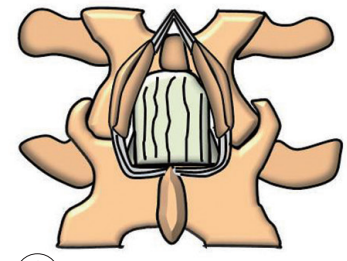

(C)

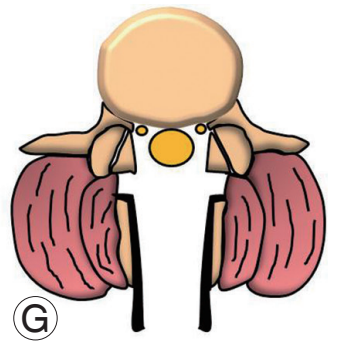

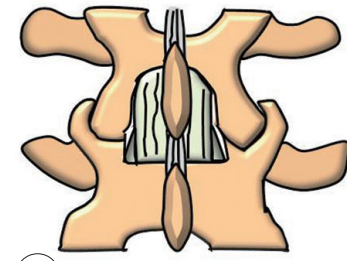

(D)

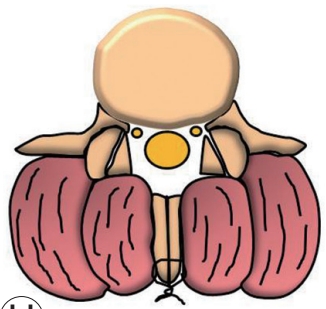

(H)

Fig. 1. Schematic diagram of the surgical procedures. (A, E) A 4-cm posterior skin incision on the midline of the spinous process. (B, F) The spinous processes were divided and the paravertebral muscles from the lamina to the medial aspect of the facet joints were exposed. (C, G) Partial laminectomy and removal of the ligamentum flavum. (D, H) Reconstruction of the split spinous process.
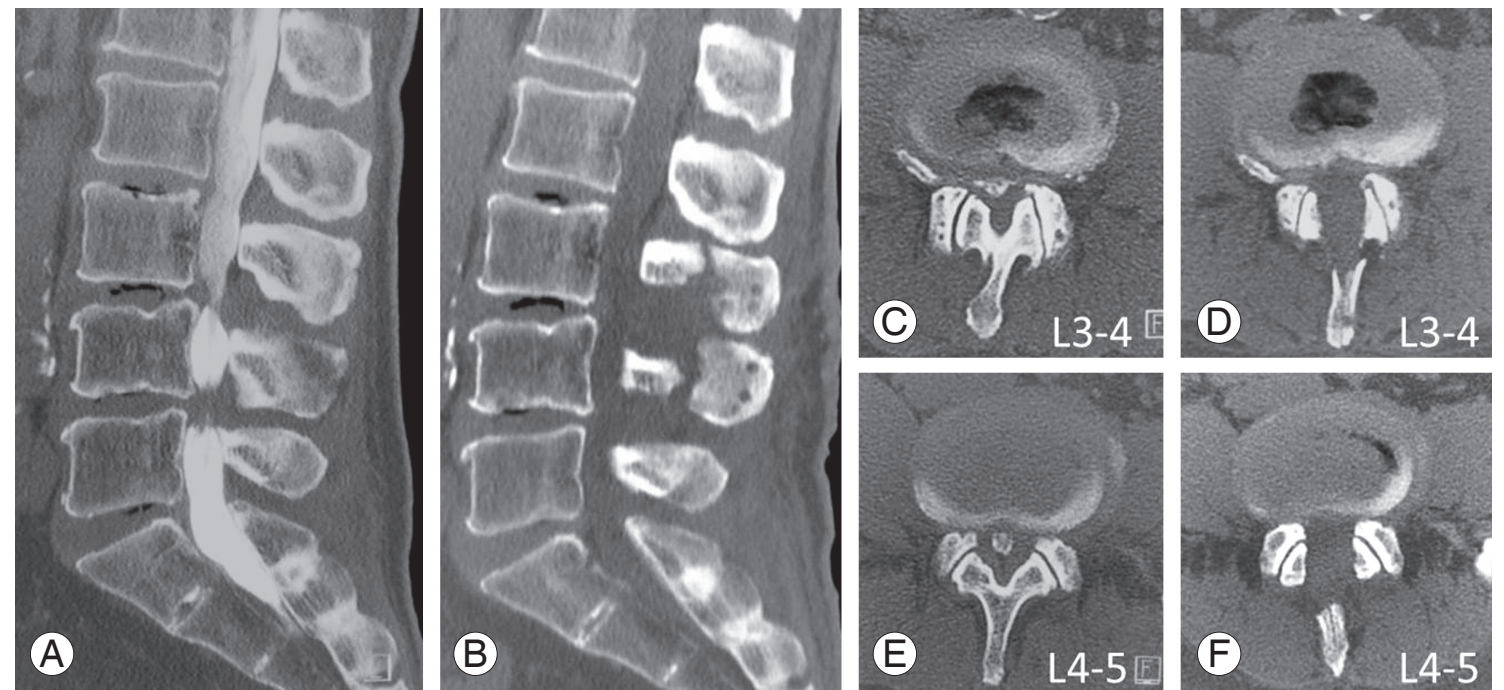

Fig. 2. In a case of 2-level decompression, preoperative computed tomography (CT)-myelography shows severe central canal stenosis at $L 3-4$ and $L 4-5(A, C, E)$, and the postoperative CT shows a reconstructed spinous process (B). Decompression has been achieved centrally with preservation of the facet joint at $L 3-4$ (D) and $L 4-5$ (F).

\section{Statistical analysis}

Continuous variables are presented as mean \pm standard deviation with ranges, and categorical variables are presented as frequencies and percentages. The independent Student $t$-test and one-way analysis of variance were used to compare two and three groups, respectively. The chisquare test or Fisher exact test were used if a frequency of any cell in a contingency table was $<5$, as appropriate. All $p$-values are two-sided, and $p$-values of $<0.05$ were con- sidered to indicate statistical significance. SPSS ver. 17.0 (SPSS Inc., Chicago, IL, USA) was used to perform statistical analyses.

\section{Results}

A total of 52 consecutive patients ( 32 males, 20 females) met the inclusion criteria and had an average follow-up of $2.6 \pm 0.8$ years (range, $2-4.5$ years). The characteristics of the patients are shown in Table 1. The average age at the 
Table 1. Patient characteristics ( $n=52)$

\begin{tabular}{lc} 
Characteristic & Value \\
\hline Age at the time of surgery (yr) & $72.1 \pm 7.1$ (61-87) \\
\hline Sex (male:female) & $32: 20$ \\
\hline Duration of follow-up (yr) & $2.6 \pm 0.8(2-4.5)$ \\
\hline Decompression level & 3 \\
\hline 1 & 27 \\
\hline 2 & 16 \\
\hline 3 & 5 \\
\hline 4 & 1 \\
\hline 5 & 19 \\
\hline Preoperative diagnosis & 19 \\
\hline Lumbar spinal stenosis & 14 \\
\hline Degenerative spondylolisthesis & \\
\hline Degenerative lumbar scoliosis & \\
\hline Values are presented as mean \pm standard deviation (range) or number.
\end{tabular}

Table 2. Summary of clinical outcomes and complications

\begin{tabular}{lc} 
Variable & \multicolumn{1}{c}{ Value } \\
JOA score & \\
\hline Preoperative & $14.6 \pm 3.7(6-21)$ \\
\hline Postoperative & $23.2 \pm 3.4(14-29)$ \\
\hline Recovery rate (\%) & $13(25)$ \\
\hline Poor clinical outcome & 2 \\
\hline Complication (dural tear) & 1 \\
\hline Reoperation & 1 \\
\hline Progression of slippage & 1 \\
\hline Far-out syndrome & \\
\hline Synovial cyst & $8 / 24(33)$ \\
\hline Radiographic outcome & $5 / 14(36)$ \\
\hline Progression of slippage & $100)$ \\
\hline Progression of curvature & \\
\hline
\end{tabular}

Values are presented as mean \pm standard deviation (range) or number (\%). JOA, Japanese Orthopedic Association.

time of surgery was $72.1 \pm 7.1$ years (range, $61-87$ years). The average number of decompression levels was $2.5 \pm 0.1$ (range, 1-5). The preoperative diagnosis consisted of LSS in 19 patients, DS in 19 patients, and DLS in 14 patients. The mean JOA score significantly increased from 14.6 \pm 3.7 to $23.2 \pm 3.4(p<0.001)$ after the operation at the last followup (Table 2). The mean recovery rate was $60.1 \% \pm 22.1 \%$ (range, $0 \%-100 \%$ ). Intraoperative and postoperative com- plications associated with LSPSL included a dural tear in two patients. Three cases required reoperation caused by progression of slippage in one case, far-out syndrome in one case, and a synovial cyst in one case. A progression of slippage $\geq 5 \mathrm{~mm}$ was found in 8 of 24 patients (33\%) in the DS group. A progression of curvature $\geq 5^{\circ}$ was found in 5 of 14 patients (36\%) in the DLS group. Among the 52 patients, 13 patients (25\%) were classified into the poor clinical outcome group (recovery rate $<50 \%$ ). None of the patient factors, including age, gender, preoperative JOA score, preoperative distance of IC, motor weakness, urinary dysfunction, duration of symptoms, and multilevel decompression were independent predictors of poor clinical outcome (Table 3 ). There was no statistically significant difference in the follow-up periods between the two groups. We compared postoperative leg pain and back pain using subscales of the JOA score between the good and poor outcome groups. The postoperative back pain was significantly worse in the poor group than in the good group ( $1.7 \pm 0.1$ vs. $2.3 \pm 0.9$, respectively; $p=0.195$ ). In contrast, the postoperative leg pain score was similar between the poor and good outcome groups ( $2.5 \pm 0.1$ vs. $2.7 \pm 0.8$, respectively; $p=0.001)$. Regarding the radiographic parameters, there was a trend towards smaller preoperative and postoperative LL in the poor outcome group relative to that of the good outcome group, although the difference was not significant (preoperative, $31.7^{\circ}$ vs. $35.5^{\circ}, p=0.249$; postoperative, $26.6^{\circ}$ vs. $32.8^{\circ}, p=0.099$, respectively). Higher rates of DLS patients were observed in the poor clinical outcome group than in the good outcome group ( $15 \%$ vs. $62 \%, p=0.003$, respectively). The preoperative and postoperative Cobb angles were greater in the poor outcome group than in the good outcome group (preoperative: $4.8^{\circ}$ vs. $10.7^{\circ}, p=0.001$; postoperative: $6.4^{\circ}$ vs. $13.7^{\circ}$, $p=0.002$, respectively). However, there was no significant difference in the change in the Cobb angle $\left(1.5^{\circ}\right.$ vs. $3.0^{\circ}$, $p=0.135)$. These results indicate that pre-existing DLS was associated with poor clinical outcome, whereas progression of the Cobb angle did not have an effect on the clinical outcome. There was no difference in the percentage of DS patients between the good and poor outcome groups ( $46 \%$ vs. $46 \%, p=1.000$ ). The preoperative percentage of slip was similar in both groups. However, there was a trend towards a progression of slip in the poor outcome group, although the difference was not significant. A progression of slippage was found in 8 of the 24 patients (33\%) with DS. A progression of scoliosis was found in 5 of the 
Table 3. Comparison of variables between the good and poor clinical outcomes groups

\begin{tabular}{|c|c|c|c|}
\hline Variable & Good group $(n=39)$ & Poor group $(n=13)$ & $p$-value \\
\hline Age $(y r)$ & $71.4 \pm 7.3$ & $74.3 \pm 6.3$ & 0.216 \\
\hline Female & $15(38)$ & $5(38)$ & 1.000 \\
\hline Motor weakness & $5(13)$ & $2(15)$ & 0.568 \\
\hline Duration of symptom (mo) & $32.6 \pm 28.4$ & $33.8 \pm 32.9$ & 0.898 \\
\hline Distance of $\mathrm{IC}(\mathrm{m})$ & $95.0 \pm 76.9$ & $99.2 \pm 126.0$ & 0.910 \\
\hline Urinary dysfunction & $10(26)$ & $4(31)$ & 0.729 \\
\hline \multicolumn{4}{|l|}{ JOA score } \\
\hline Preoperative & $14.7 \pm 3.6$ & $14.5 \pm 3.9$ & 0.864 \\
\hline Postoperative & $24.6 \pm 2.3$ & $19.1 \pm 2.9$ & $<0.001^{\text {a) }}$ \\
\hline Recovery rate $(\%)$ & $69.8 \pm 13.8$ & $31.0 \pm 16.2$ & $<0.001^{\text {a) }}$ \\
\hline Follow-up period (yr) & $2.6 \pm 0.8$ & $2.7 \pm 0.8$ & 0.505 \\
\hline \multicolumn{4}{|l|}{ Lumbar lordosis $\left({ }^{\circ}\right)$} \\
\hline Preoperative & $35.5 \pm 10.8$ & $31.7 \pm 8.3$ & 0.249 \\
\hline Postoperative & $32.8 \pm 12.2$ & $26.6 \pm 607$ & 0.099 \\
\hline Degenerative lumbar scoliosis $\left({ }^{\circ}\right)$ & $6(15)$ & $8(62)$ & $0.003^{\mathrm{a})}$ \\
\hline Preoperative Cobb & $4.8 \pm 4.5$ & $10.7 \pm 6.5$ & $0.001^{a)}$ \\
\hline Postoperative Cobb & $6.4 \pm 6.1$ & $13.7 \pm 9.7$ & $0.002^{a)}$ \\
\hline$\triangle$ Cobb & $1.5 \pm 2.3$ & $3.0 \pm 4.6$ & 0.135 \\
\hline Degenerative spondylolisthesis (\%) & $18(46)$ & $6(46)$ & 1.000 \\
\hline Preoperative slip & $5.3 \pm 7.2$ & $7.7 \pm 8.4$ & 0.331 \\
\hline Postoperative slip & $6.5 \pm 8.4$ & $11.1 \pm 11.0$ & 0.155 \\
\hline$\Delta$ Slip & $1.2 \pm 2.1$ & $3.5 \pm 4.8$ & 0.116 \\
\hline
\end{tabular}

Values are presented as mean \pm standard deviation or number (\%).

IC, intermittent claudication; JOA, Japanese Orthopedic Association.

a) Statistically significant.

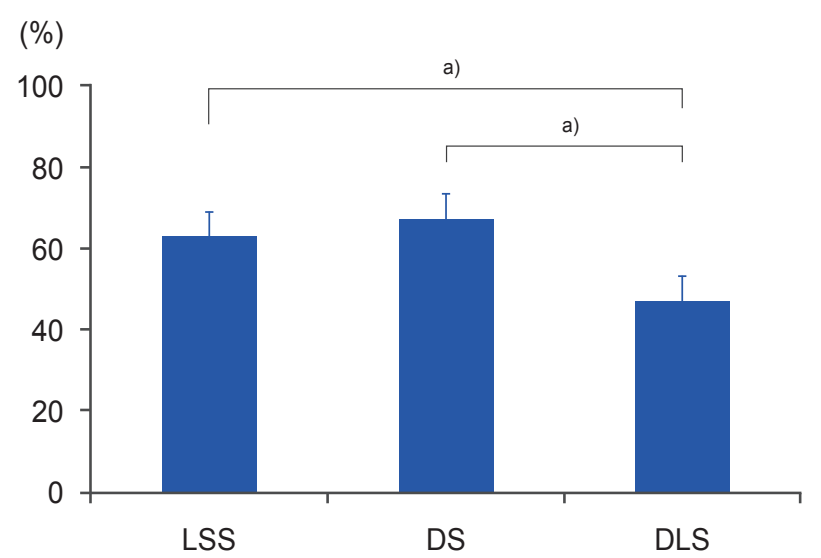

Fig. 3. Comparison of recovery rates among the lumbar spinal stenosis (LSS), degenerative spondylolisthesis (DS), and degenerative lumbar scoliosis (DLS) groups. The recovery rate was significantly lower in the DLS group than in the LSS and the DS groups (LSS group, 62.8\%; DS group, 67.1\%; DLS group, 46.9\%). ${ }^{\text {a) }}$ Statistically significant.
14 patients (36\%) with DLS. The recovery rate was significantly lower in the DLS group than in the LSS and DS groups (DLS group, 46.9\%; LSS group, 62.8\%; DS group, $67.1 \%$ ) (Fig. 3). There was no significant difference in the three groups (LSS, DS, DLS) with respect to age (70.6 \pm 7.3 , $71.6 \pm 7.2,75.0 \pm 6.4$ years, respectively; $p=0.204)$, sex $(20 \%$, $40 \%, 40 \%$, respectively; $p=0.100$ ), number of decompression levels $(2.5 \pm 0.9,2.4 \pm 0.7,2.6 \pm 0.9$, respectively; $p=0.642)$, and preoperative JOA score $(14.0 \pm 3.7,15.3 \pm 3.5$, $14.6 \pm 3.7$, respectively; $p=0.527)$. These results suggested that only pre-existing DLS was significantly associated with poor clinical outcome.

\section{Discussion}

Degenerative LSS is the most common cause of lumbar spine surgery in adults [10]. Recent prospective random- 
ized studies with long-term follow-up have demonstrated that surgical treatment is superior to nonsurgical treatment for degenerative LSS [2]. In the current study, with a mean follow-up of 2.6 years, the overall mean JOA score significantly improved, and the recovery rate was $60 \%$ after LSPSL. The results of this study indicate that the clinical outcomes of LSPSL for LSS were favorable. Conventional laminectomy has been reported as having a potential risk of iatrogenic instability and back muscle damage [3-5]. Johnsson et al. [3] reported that $40 \%$ of patients showed progressive slippage postoperatively with conventional laminectomy. Less invasive decompression procedures, including microscopic or microendoscopic lumbar decompression via a unilateral approach, have been reported as giving satisfactory clinical outcomes for the treatment of LSS [11-15]. However, these procedures are technically demanding, and the working space is limited, particularly in cases of upper lumbar lesions. LSPSL has been reported to reduce muscle damage and postoperative wound pain in the treatment of LSS [8,16-18]. The advantages of this procedure include obtaining a wider surgical working space with less invasive muscle damage and the possible prevention of excessive removal of the facet joint. In our study, the radiographic outcomes after LSPSL in the LSS and DS groups were equivalent to those of conventional laminectomy [4]. The progression of spillage after LSPSL did not influence clinical outcomes and reoperation. These results, which are based on an average follow-up of 2.6 years, suggest that LSPSL is an effective less invasive surgical treatment for patients with LSS or DS. LSPSL has been increasingly used as an alternative to lumbar laminectomy with some modifications [19-21].

We found that patients with pre-existing DLS were associated with worse clinical outcomes after surgical treatment. Kelleher et al. [22] compared the clinical outcomes of minimally invasive decompression for LSS in patients with and without deformities. They concluded that DLS patients, particularly those with lateral listhesis, had a significantly higher rate of revision surgery $(25 \%)$ than LSS patients (0\%) and DS patients (4\%). Toyoda et al. [14] reported 5-year and longer clinical outcomes in patients treated with microsurgical decompression for LSS, DS, and DLS, finding no significant differences in the postoperative JOA scores at the final follow-up. However, a higher rate of reoperation was found in the DLS group (25\%) than in the LSS group (0\%) and DS group (10\%) [14]. Decompression with fusion surgery for patients with DLS is still controversial. A previous study suggested that decompression and fusion surgery prevent revision surgery better than decompression alone in the setting of mild degenerative scoliosis $[23,24]$. A higher rate of recurrence of symptoms was observed in patients who underwent decompression alone (75\%) than in those who underwent decompression and fusion (34\%) during a 5-year followup [23]. Progression of scoliosis was found in $36 \%$ of patients with DLS, a percentage similar to that in all patients with DLS in Japan [24].

There were several limitations in this study, which include the relatively small sample size and retrospective data collection. In addition, the postoperative follow-up period may not have been long enough to assess the potential risk of iatrogenic instability. Recently, a prospective randomized study demonstrated that clinical outcomes and back pain were similar between LSPSL and conventional techniques [25]. A prospective randomized study with a larger sample size and a longer follow-up period would be required to thoroughly assess the clinical outcomes after LSPSL. Furthermore, we did not evaluate the preoperative global spinal alignment for most of these patients. In recent literature, sagittal global alignment and pelvic parameters were shown to correlate with healthrelated quality of life scores [26]. In the current study, there was a trend towards smaller preoperative and postoperative LL in the poor outcome group. There is some possibility that the global spinal alignment was worse in the poor outcome group than in the good outcome group and may have influenced the clinical outcomes.

\section{Conclusions}

The results of this study showed that the clinical and radiographic outcomes of LSPSL for LSS and DS were favorable. Progression of slippage and scoliosis were not shown to affect the clinical outcomes. However, progression of slippage was found in $33 \%$ of the DS group with average 2.6-year follow-up. A long-term follow-up study for DS after LSPSL is needed. Pre-existing DLS was a significant risk factor associated with poor clinical outcomes. Appropriate patient selection with consideration to decompression surgery may be important for DLS patients.

\section{Conflict of Interest}

No potential conflict of interest relevant to this article was 
reported.

\section{References}

1. Weinstein JN, Tosteson TD, Lurie JD, et al. Surgical versus nonsurgical therapy for lumbar spinal stenosis. N Engl J Med 2008;358:794-810.

2. Lurie JD, Tosteson TD, Tosteson A, et al. Long-term outcomes of lumbar spinal stenosis: eight-year results of the Spine Patient Outcomes Research Trial (SPORT). Spine (Phila Pa 1976) 2015;40:63-76.

3. Johnsson KE, Willner S, Johnsson K. Postoperative instability after decompression for lumbar spinal stenosis. Spine (Phila Pa 1976) 1986;11:107-10.

4. Katz JN, Lipson SJ, Chang LC, Levine SA, Fossel AH, Liang MH. Seven- to 10-year outcome of decompressive surgery for degenerative lumbar spinal stenosis. Spine (Phila Pa 1976) 1996;21:92-8.

5. Mardjetko SM, Connolly PJ, Shott S. Degenerative lumbar spondylolisthesis: a meta-analysis of literature 1970-1993. Spine (Phila Pa 1976) 1994;19(20 Suppl):2256S-2265S.

6. Gelalis ID, Stafilas KS, Korompilias AV, Zacharis KC, Beris AE, Xenakis TA. Decompressive surgery for degenerative lumbar spinal stenosis: long-term results. Int Orthop 2006;30:59-63.

7. Yang JC, Kim SG, Kim TW, Park KH. Analysis of factors contributing to postoperative spinal instability after lumbar decompression for spinal stenosis. Korean J Spine 2013;10:149-54.

8. Watanabe K, Hosoya T, Shiraishi T, Matsumoto M, Chiba K, Toyama Y. Lumbar spinous process-splitting laminectomy for lumbar canal stenosis: technical note. J Neurosurg Spine 2005;3:405-8.

9. Hirabayashi K, Miyakawa J, Satomi K, Maruyama T, Wakano K. Operative results and postoperative progression of ossification among patients with ossification of cervical posterior longitudinal ligament. Spine (Phila Pa 1976) 1981;6:354-64.

10. Deyo RA, Gray DT, Kreuter W, Mirza S, Martin BI. United States trends in lumbar fusion surgery for degenerative conditions. Spine (Phila Pa 1976) 2005;30:1441-5.

11. Arai $Y$, Hirai T, Yoshii T, et al. A prospective comparative study of 2 minimally invasive decompression procedures for lumbar spinal canal stenosis: unilateral laminotomy for bilateral decompression (ULBD) versus muscle-preserving interlaminar decompression (MILD). Spine (Phila Pa 1976) 2014;39:332-40.

12. Mobbs RJ, Li J, Sivabalan P, Raley D, Rao PJ. Outcomes after decompressive laminectomy for lumbar spinal stenosis: comparison between minimally invasive unilateral laminectomy for bilateral decompression and open laminectomy: clinical article. J Neurosurg Spine 2014;21:179-86.

13. Pao JL, Chen WC, Chen PQ. Clinical outcomes of microendoscopic decompressive laminotomy for degenerative lumbar spinal stenosis. Eur Spine J 2009; 18:672-8.

14. Toyoda H, Nakamura H, Konishi S, Dohzono S, Kato M, Matsuda H. Clinical outcome of microsurgical bilateral decompression via unilateral approach for lumbar canal stenosis: minimum five-year follow-up. Spine (Phila Pa 1976) 2011;36:410-5.

15. Minamide A, Yoshida M, Yamada H. Endoscopeassisted spinal decompression surgery for lumbar spinal stenosis. J Neurosurg Spine 2013;19:664-71.

16. Watanabe K, Matsumoto M, Ikegami T. Reduced postoperative wound pain after lumbar spinous process-splitting laminectomy for lumbar canal stenosis: a randomized controlled study. J Neurosurg Spine 2011;14:51-8.

17. Uehara M, Takahashi J, Hashidate H. Comparison of spinous process-splitting laminectomy versus conventional laminectomy for lumbar spinal stenosis. Asian Spine J 2014;8:768-76.

18. Lee DY, Lee SH. Spinous process splitting laminectomy for lumbar canal stenosis: a critical appraisal. Minim Invasive Neurosurg 2008;51:204-7.

19. Nomura H, Yanagisawa Y, Arima J, Oga M. Clinical outcome of microscopic lumbar spinous processsplitting laminectomy: clinical article. J Neurosurg Spine 2014;21:187-94.

20. Kanbara S,Yukawa Y, Ito K, Machino M, Kato F. Surgical outcomes of modified lumbar spinous processsplitting laminectomy for lumbar spinal stenosis. J Neurosurg Spine 2015;22:353-7.

21. Henky J, Yasuda M, Arifin MZ, Takayasu M, Faried A. Trumpet laminectomy microdecompression for lumbal canal stenosis. Asian Spine J 2014;8:667-74.

22. Kelleher MO, Timlin M, Persaud O, Rampersaud YR. Success and failure of minimally invasive decompression for focal lumbar spinal stenosis in patients with and without deformity. Spine (Phila Pa 1976) 2010; 
35:E981-7.

23. Daubs MD, Lenke LG, Bridwell KH, Cheh G, Kim YJ, Stobbs G. Decompression alone versus decompression with limited fusion for treatment of degenerative lumbar scoliosis in the elderly patient. Evid Based Spine Care J 2012;3:27-32.

24. Jimbo S, Kobayashi T, Aono K, Atsuta Y, Matsuno T. Epidemiology of degenerative lumbar scoliosis: a community-based cohort study. Spine (Phila Pa 1976) 2012;37:1763-70.
25. Rajasekaran S, Thomas A, Kanna RM, Prasad Shetty A. Lumbar spinous process splitting decompression provides equivalent outcomes to conventional midline decompression in degenerative lumbar canal stenosis: a prospective, randomized controlled study of 51 patients. Spine (Phila Pa 1976) 2013;38:1737-43.

26. Schwab F, Ungar B, Blondel B. Scoliosis Research Society-Schwab adult spinal deformity classification: a validation study. Spine (Phila Pa 1976) 2012;37:107782. 